\title{
3 Research Square

\section{Crucial Conversations for High Risk Populations Before Surgery: Advance Care Planning in a Preoperative Setting}

Roma Gautam Patel ( $\sim$ roma.g.patel@gmail.com )

Indiana University Health https://orcid.org/0000-0001-7586-4303

Alexia Torke

Indiana University Health

Barb Nation

Indiana University School of Medicine

Ann Cottingham

Indiana University School of Medicine

Jennifer Hur

Indiana University School of Medicine

Rachel Gruber

Indiana University School of Medicine

Shilpee Sinha

Indiana University School of Medicine

Research

Keywords: advance care planning, mortality, readmissions, pre-operative clinic

Posted Date: November 5th, 2020

DOI: https://doi.org/10.21203/rs.3.rs-100950/v1

License: (c) (1) This work is licensed under a Creative Commons Attribution 4.0 International License.

Read Full License

Version of Record: A version of this preprint was published at Palliative Medicine Reports on October 1st, 2021. See the published version at https://doi.org/10.1089/pmr.2021.0015. 


\section{Abstract}

Background: High-risk patients undergoing elective surgery may experience life-threatening complications. Preoperative assessment clinics provide an opportunity to conduct Advance Care Planning but it is unknown how often this is accomplished or subsequently needed for goals of care discussions.

Objective: 1) assess the relationship between advance directives with readmissions and mortality at one year 2) qualitatively examine clinical events that occurred for patients who died during follow-up.

Design: This was an observational cohort study conducted via chart review. Patients were followed for one year.

Participants: Four hundred patients who were undergoing preoperative evaluation for elective surgery at two academic hospitals.

Main measures: The prevalence of advance directives at the time of surgery, prevalence of advance directives in the electronic medical record during the one year follow-up period, readmissions and mortality at one year.

Key Results: Three hundred and ninety patients were included. There were 102 (26.4\%) patients were readmitted, which was not associated with having an AD on file. Seventeen (4.4\%) filed an AD during follow-up. Twelve of 19 (63\%) patients who died had an AD on file at the time of death. There was a significant association between having an $A D$ at any time with mortality (chi-square $p$-value $<0.001$ ). Total mortality for the cohort was $4.9 \%$. Of the 19 patients who died, seven (37\%) underwent resuscitation, four of whom had an $A D$ on file.

Conclusions: A minority of patients who die within a year after major surgery have an AD, highlighting the missed opportunity to conduct advance care planning in a preoperative clinic.

\section{Introduction}

Factors such as age and chronic disease are associated with high surgical risk ${ }^{1,2}$. High-risk patients who undergo elective surgery have an increased likelihood of life-threatening complications during the perioperative period ${ }^{3}$. Such patients may also be at high risk of other medical complications even after the surgery. The profound impact of the novel coronavirus-19 in the population exemplifies the importance of $A D$ completion.

Preoperative clinics evaluate patients prior to surgery and are shown to decrease operative room cancellations, post-operative length of stay and possibly mortality ${ }^{4,5}$. Some preoperative clinics employ internal medicine certified hospitalists to provide nuanced risk assessments and medically optimize patients prior to surgery. These clinics are potential venues to discuss Advance Care Planning (ACP) as patients seen in preoperative clinics are already being advised of their comorbidity and procedurally 
associated risks. Counselling and documentation of Advance Directives (ADs) is not standard at these clinics, though prior research has demonstrated that it is feasible, even within clinic time constraints ${ }^{6}$.

Studies have estimated the community AD completion rate to be between $5-25 \% 6,7$. In one study of intensive care unit patients, $42 \%$ of patients had an $A D$, however the cohort was known to be significantly ill, with a mortality of $24 \%^{8}$. In contrast, another study examining the prevalence of ADs in a cohort of cancer patients undergoing surgery consultation reported $10 \%$ of these patients had a Do-Not-Resuscitate order in their medical record but none had an $A D$ on file ${ }^{9}$. Studies have also demonstrated completion of $A D s$ are associated with lower readmission rates ${ }^{10,11}$, which have implications for both individual patients and health systems.

We have previously published a retrospective chart review of patients seen in a preoperative clinic to identify the prevalence of $A D s$, including living wills or surrogate decision maker (SDM) documentation ${ }^{12}$. In the present analysis, we followed these patients for one year post-operatively to evaluate for additional medical events that are often discussed during ACP. The primary objective was to determine if patients who had either readmission or death at one year were more likely to have ADs. The secondary objective was to qualitatively examine the events that occurred for patients who died during follow-up.

\section{Methods}

This study was an observational cohort study conducted at two, large, urban, academic hospitals via chart review. Four hundred consecutive patients between February-March 2017 undergoing preoperative evaluation for elective surgery at two Pre-Admission Testing (PAT) Clinics were identified. This study was approved by the Indiana University Institutional Review Board.

The PAT clinic appointment usually occurred days to weeks prior to surgery. Demographics and comorbidities were obtained from the preoperative evaluation in the Electronic Medical Record (EMR). The EMR was then reviewed at one year from the PAT clinic appointment date for the presence of ADs, mortality, and readmissions. Patients were coded as having an AD on file if they had either an SDM, living will or any other type of AD document in the EMR. Statistical methods included descriptive statistics of patient characteristics, and chi-square and Fisher's exact tests for comparison between groups.

\section{Results}

A total of 400 charts were reviewed. At one-year follow-up, ten patients did not undergo surgery, thus 390 records were included. The average age of patients was 58.4 years (SD 14.6) and 187 (48\%) were male. The cohort was predominantly white (85\%), with $14 \%$ African American. There was an even split of patients seen at the two clinics (51\% versus $49 \%$ ). Forty-two (10.8\%) patients had Medicaid insurance, 183 (47\%) had Medicare, 144 (37\%) had private insurance - though some patients had a combination. Only 63 (16.2\%) had an AD in the EMR prior to surgery. A previously published article discusses the comorbidities, functional status, and pre-operative risk in this patient population ${ }^{12}$. 
There were 102 (26.4\%) patients readmitted at least once. Readmissions were not associated with having an $A D$ on file at follow-up ( $p$-value 0.42). About one-third of the readmissions were with patients with underlying cancer, the majority of whom underwent surgery related to their malignancy. An additional 40 $(10.3 \%)$ of patients returned to the Emergency Department during follow-up but were not admitted.

Seventeen (4.4\%) patients filed an AD during follow-up, two of whom also had an AD on file at the time of surgery. Of the 15 patients who had no $A D$ s on file prior to surgery and completed one during follow-up, nine died. Twelve of $19(63 \%)$ patients who died had an AD on file at the time of death (Fig. 1). There was a significant association between having an $A D$ at any time with mortality ( $18 \%$ of survivors compared with $63 \%$ of decedents, chi-square $p$-value $<0.001)$. A total of three of the decedents $(17 \%)$ had an $A D$ on file prior to surgery.

Three patients died during the index hospitalization and 16 patients died during the follow-up year, for a total mortality of $4.9 \%$. The 19 patients who died had an average age of 58.5 years (SD 14.3 years) and an average Charlson Risk Score of 4.6 (SD 3.0, range 2 to 11). Fourteen of the 19 patients had an underlying diagnosis of cancer; four of these patients had known metastatic cancer. Of these, 13 underwent surgery for resection of their cancer and one had surgery for a pathologic fracture.

Three of the surviving patients required resuscitation during their initial hospitalization. One suffered an MCA stroke and thus required re-intubation. Another patient required re-intubation due to respiratory distress and ultimately required a tracheostomy prior to discharge. The third patient required re-intubation and vasopressors, thought to be due to complications from their surgery. Of the 19 patients who died, seven (37\%) underwent resuscitation - three of whom were intubated and four of whom experienced cardiopulmonary arrest. Four of the seven patients who underwent resuscitation had an AD on file (two had an SDM and two had a living will). Five of the seven patients had return of spontaneous circulation (ROSC); the other two died during the attempted resuscitation. The five patients who had ROSC all were ultimately transitioned to comfort measures.

\section{Discussion And Conclusions}

Similar to prior studies, in this chart review, approximately $15 \%$ of patients had an AD on file $e^{8,9}$. Over a quarter of the patients were readmitted in the following year. Our data does not find an association between readmission and having an $A D$ on file, which was surprising as readmitted patients have more contacts with the health system and are potentially a sicker portion of the cohort. This is equally surprising considering one-third of our readmitted patients had a diagnosis of potentially lethal cancer.

Our mortality rate was $<5 \%$. Other studies of patients undergoing elective surgeries had mortality rates ranging from $<1 \%$ to approximately $3 \%$, however these studies examined in-hospital and/or six month mortality $1,2,5$. The overall low mortality rate may be more of a reflection of the safety of modern surgery rather than improved health of our patients. Recent data suggests that patient frailty, which takes medical co-morbidities as well as cognitive and functional measures into account, may be a more powerful risk 
factor for post-operative outcomes ${ }^{13,14}$. Frailty may be a better marker to identify which patients may benefit most from $A D$ completion prior to surgery.

Only $17 \%$ of patients who died in the cohort had an $A D$ on file prior to surgery and $63 \%$ of patients who died had an AD on file prior to death. While there was a statistically significant relationship between having an $A D$ on file and death, some of those were likely in response to the patient's declining condition. Further review revealed that patients who died were high risk based on having cancer and other advanced diseases at the time of surgery. These findings suggest a missed opportunity for identifying patients with high one-year mortality risk so that ACP conversations can occur and ADs can be completed in advance.

Almost $40 \%$ of patients who died were resuscitated prior to their death. The perioperative evaluation is a missed opportunity to explore whether life sustaining treatments were consistent with the patient's goals. Studies have demonstrated that the more elements of goals of care that are discussed, the better agreement between patient's preferences and goals of care ${ }^{15}$. Moreover, ACP is correlated with decreased aggressive measures at the end of life and increased concordance with patients' end of life wishes ${ }^{16}$.

There are several limitations to this study. It was conducted within one health system, including one tertiary referral center, which may attract a higher risk surgical population. Data was collected over only one year and was observational. Finally, the high percentage of surgeries performed for cancer affected the likelihood of patients proceeding to surgery with minimal discussion as most preoperative clinics are more likely to recommend proceeding to cancer surgery without delay than for elective procedures.

In conclusion, the finding that a minority of patients who die within a year of major surgery have an advance directive highlights a missed opportunity to conduct advance care planning in a perioperative clinic. Future studies should include the patient perspective on how patients undergoing elective procedures would have benefited from the opportunity to discuss advance care planning along with the implementation of advance care planning in preoperative clinics and subsequent measure of effect on patient expectations and experience.

\section{Declarations}

\section{Funding}

There was no sources of funding for this study.

\section{Authors' contributions}

AT and SS designed the project, BN, AC, RG, SS, and RP performed acquisition and analysis, RP, AT, JR, and SS interpreted the data, RP drafted the manuscript and all authors contributed to substantially revising the manuscript. 


\section{Acknowledgements}

Not applicable

\section{References}

1. Berry AJ, lii RBS, Weintraub WS, et al. Age versus comorbidities as risk factors for complications after elective abdominal aortic reconstructive surgery. 1996:345-352. doi:10.1067/mva.2001.111737

2. Naughton C, Feneck RO. The impact of age on 6-month survival in patients with cardiovascular risk factors undergoing elective non-cardiac surgery. 2007;(May):768-776. doi:10.1111/j.17421241.2007.01304.x

3. Nelson MT, Spencer CC, Thompson A. 2014 ACC / AHA Guideline on Perioperative Cardiovascular Evaluation and Management of Patients Undergoing Noncardiac Surgery. 2014;64(22). doi:10.1016/j.jacc.2014.07.944

4. van Klei WA, Moons KG, Rutten CL, et al. The Effect of Outpatient Preoperative Evaluation of Hospital Inpatients on Cancellation of Surgery and Length of Hospital Stay. 2002:644-649.

5. Blitz JD, Kendale SM, Jain SK, Cuff GE, Kim JT, Rosenberg AD. Preoperative Evaluation Clinic Visit Is Associated with Decreased Risk of In-hospital Postoperative Mortality. 2017;(2):280-294.

6. Grimaldo DA, Wiener-kronish JP, Jurson T, Shaughnessy TE, Curtis JR, Liu LL. A Randomized, Controlled Trial of Advance Care Planning Discussions during Preoperative Evaluations. 2001;(1):4350 .

7. Rao JK, Anderson LA, Lin F, et al. Completion of Advance Directives Among U.S. Consumers. 2015;46(1):65-70. doi:10.1016/j.amepre.2013.09.008.Completion

8. Gamertsfelder EM, Seaman JB, Tate J, Buddadhumaruk P, Happ MB. Prevalence of Advance Directives among Older Adults Admitted to Intensive Care Units and Requiring Mechanical Ventilation. 2019;42(4):34-41. doi:10.3928/00989134-20151124-02.Prevalence

9. Marcia L, Ashman ZW, Pillado EB, Kim DY. Advance Directive and Do-Not-Resuscitate Status among Advanced Cancer Patients with Acute Care Surgical Consultation. 2014:1565-1570.

10. J M, BA S. Integration of an Advance Care Planning Model in Home Health: Favorable Outcomes in End-of-Life Discussions, POLST Rates, and 60-day Hospital Readmissions. Home Heal Now. 2019;37(6):337-344.

11. Bond WF, Kim M, Franciskovich CM, Al BET. Advance Care Planning in an Accountable Care Organization Is Associated with Increased Advanced Directive Documentation and Decreased Costs. J Palliat Med. 2018;21(4). doi:10.1089/jpm.2017.0566

12. Sinha S, Gruber RN, Cottingham AH, et al. Advance Care Planning in A Preoperative Clinic: A Retrospective Chart Review. 2019:523-525. doi:10.1007/s11606-018-4744-8

13. Mrdutt MM, Papaconstantinou HT, Robinson BD, Bird ET, Isbell CL. Preoperative Frailty and Surgical Outcomes Across Diverse Surgical Subspecialties in a Large Health Care System. J Am Coll Surg. 
2019;228(4):482-490. doi:10.1016/j.jamcollsurg.2018.12.036

14. Mclsaac DI, Taljaard M, Bryson GL et al. Frailty as a Predictor of Death or New Disability After Surgery. Ann Surg. 2020;271:283-289.

15. You J, Dodek P, Lamontagne F, et al. What really matters in end-of-life discussions? Perspectives of patients in hospital with serious illness and their families. CMAJ. 2014;186(18):679-687.

16. Brinkman-stoppelenburg A, Rietjens JAC, Heide A Van Der. The effects of advance care planning on end-of-life care: A systematic review. Palliat Med. 2014;28(8):1000-1025.

doi:10.1177/0269216314526272

\section{Tables}

Characteristics and outcomes of survivors and decedents. 


\begin{tabular}{|lll|}
\hline Variable & Survivors & Decedents N = 19, \\
& $\mathrm{N}=371$, & Number (\%) \\
& Number (\%) & \\
\hline Gender & & \\
Female & $194(52)$ & $9(47)$ \\
\hline Surgery & & \\
Cancer & $102(28)$ & $14(74)$ \\
Dental & $16(4)$ & $2(11)$ \\
General surgery & $46(12)$ & $0(0)$ \\
Ear, nose and throat & $34(9)$ & $0(0)$ \\
Gynecology & $11(3)$ & $0(0)$ \\
Neurosurgery & $61(16)$ & $0(0)$ \\
Orthopedic & $53(14)$ & $1(5)$ \\
Urology & $12(3)$ & $0(0)$ \\
Other & $36(10)$ & $2(11)$ \\
\hline Cancer diagnosis, total & 104 & 15 \\
Metastatic & $21(20)$ & $4(27)$ \\
Not metastatic or unknown & $83(80)$ & $11(73)$ \\
\hline Palliative Care consulted & $5(1)$ & $2(11)$ \\
\hline Died during initial/surgical hospitalization & N/A & $3(16)$ \\
\hline Died in the hospital & N/A & $8(42)$ \\
\hline Died in hospice & N/A & $3(16)$ \\
Resuscitation (CPR or intubation) & $3(1)$ & $7(37)$ \\
Died during code & N/A & $2(29)$ \\
\hline
\end{tabular}

\section{Figures}




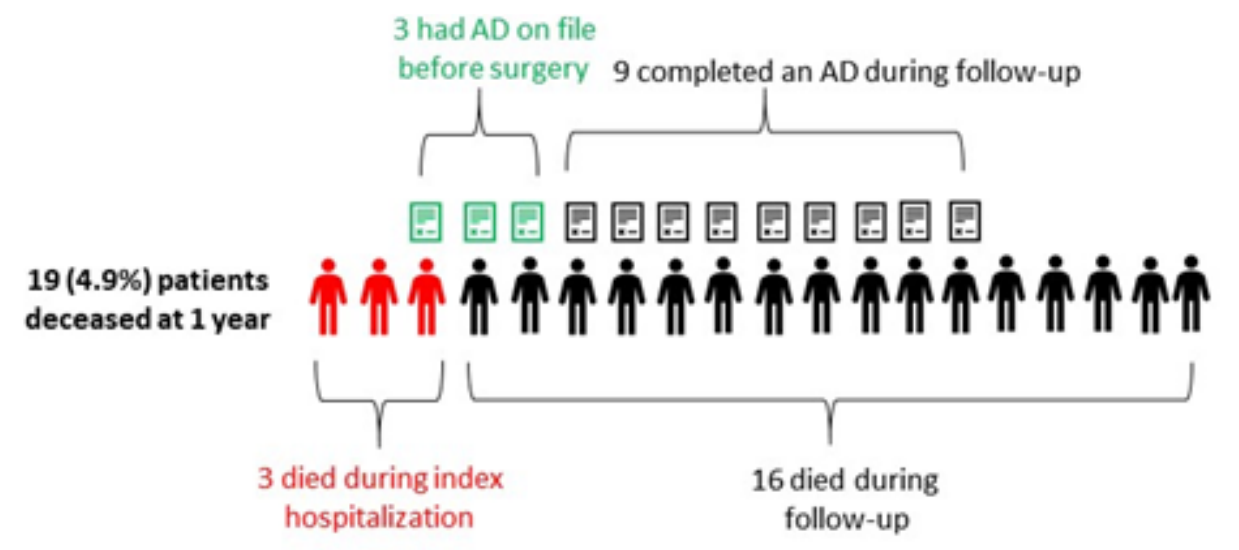

Figure 1

Mortality data 